Barrera-Algarín, E., Sarasola-Sánchez-Serrano, J.L., Fernández-Reyes, T. y García-González, Abraham (2021). Déficit en la formación sobre altas capacidades de egresados en Magisterio y Pedagogía: Un hándicap para la Educación primaria en Andalucía. Revista de Investigación Educativa, 39(1), 209-226.

DOI: http://dx.doi.org/10.6018/rie.422431

\title{
Déficit en la formación sobre altas capacidades de egresados en Magisterio y Pedagogía: Un hándicap para la Educación primaria en Andalucía
}

\author{
Deficit in Teachers and Educators' Training on Intellectual \\ Giftedness:A Handicap for Elementary Education in Andalusia
}

\author{
Evaristo Barrera-Algarín*, José Luís Sarasola-Sánchez-Serrano*,Teresa Fernández-Reyes** y \\ Abraham García-González* \\ * Universidad Pablo de Olavide \\ ** Centro para niños y niñas con altas capacidades intelectuales, superdotados y talentos (CADIS)
}

\begin{abstract}
Resumen
El objetivo de la investigación fue analizar el nivel de formación que el profesorado tiene a su disposición en temas de altas capacidades desde su formación en Grado y Posgrados en Andalucía. Hipótesis de partida: El profesorado no cuenta con suficiente formación de grado y posgrado para poder trabajar en el aula las necesidades educativas especiales de los menores con altas capacidades. Teniendo en cuenta este objetivo e hipótesis, se llevó a cabo un análisis de los planes de estudios universitarios de grado y posgrado vinculados a las altas capacidades en las universidades públicas y privadas de Andalucía. Metodología: Análisis documental, utilizando información oficial, sobre formación de grado y posgrado en altas capacidades a través de 5 variables, en todas las universidades públicas de Andalucía, más la UNED, en titulaciones de Grados vinculados a las plantillas de referencia de los Centros educativos de primaria de Andalucía: 35 asignaturas de grado, y un total de 184 temas específicos o módulos específicos de contenido en referencia a Necesidades Educativas Especiales o altas capacidades. También se realizó estudio de 8 Títulos de posgrados, y de la oferta privada nacional. Principales resultados: La media andaluza en formación de grado sobre altas capacidades es tan sólo de $0.63 \%$ de
\end{abstract}

Correspondencia: Evaristo Barrera-Algarín, ebaralg@upo.es, Universidad Pablo de Olavide, Ctra. de Utrera, 1, 41013, Sevilla 
créditos optativos (37.72 horas) y 0.22\% de créditos obligatorios (13.07 horas) en materia de altas capacidades. En posgrado, estos no se centran exclusivamente en altas capacidades, siendo la media de créditos empleados en formar al estudiante de $1.35 \%$ respecto al total del posgrado.

Palabras clave: Altas Capacidades; Profesorado; Educación Primaria; Formación de grados; Formación en Posgrados.

\section{Abstract}

The aim of this study was to analyze the training on giftedness that teachers receive in their undergraduate and graduate degrees in Andalusia. Hypothesis: Teachers do not have sufficient undergraduate and graduate training to be able to work in the classroom with the special educational needs of gifted children. Based on our objective and hypothesis, we have carried out an analysis of the undergraduate and graduate university curricula as regards giftedness in the public universities of Andalusia. Methodology: Documentary analysis using public and official information of undergraduate and graduate programs about gifted education, by means of 5 variables, and in all the public universities of Andalusia and the UNED, in degrees which followed the reference educational model of Andalusia: 35 undergraduate subjects, and 184 specific topics or modules on gifted children. Eight graduate courses were also studied, as well as the national private offer. Results: The Andalusian average for undergraduate training on giftedness is only $0.63 \%$ of optional credits (37.72 hours) and $0.22 \%$ of compulsory credits (13.07 hours). In graduate studies, these credits are not exclusively focused on giftedness, with the average number of credits used in training students being $1.35 \%$ of the total for the graduate course.

Keywords: Gifted; Teachers; Elementary School; Graduate Education; Postgraduate Education.

\section{Introducción}

Es indudable la evolución terminológica y de estudios que en las últimas décadas han intentado definir y delimitar las altas capacidades y sus tipologías. Las aportaciones de Renzulli (2016), Coleman y Cross (2005) y Sternberg y Davidson (2005) nos dan el marco de comprensión de lo que entendemos por altas capacidades, y la superdotación. La superdotación precisamente es entendida como la posesión de tres conjuntos básicos de características estrechamente relacionadas y con un igual énfasis en cada una de ellas: Una capacidad intelectual superior a la media, en relación tanto a habilidades generales como específicas; un alto grado de dedicación a las tareas refiriéndose a perseverancia, resistencia, práctica dedicada, confianza en sí mismo, etc.; y altos niveles de creatividad, "considerando la creatividad como capacidad de las personas para responder con fluidez, flexibilidad y originalidad"(Barrera, Durán, González, \& Reina, 2008, p. 9).

Todo parte de lo que se entienda por inteligencia y de cómo se pueda medir. Hoy día la base de esta conceptuialización se sustenta en las aportaciones que hiciera Gardner (1998), pero la corriente de Gardner aunque es la más extendida en el mundo educativo, no es ni la única ni la más actual sobre la Teoría de las Inteligencias Múltiples; también se podría acudir a fuentes como las de Subotnik, Olszewski-Kubilius y Worrell (2019). 
En cualquier caso, la inteligencia de tipo académica (resultados académicos), no es suficiente para medir la inteligencia de un sujeto, ya que estamos compuestos por varias inteligencias que nos conforman. Si el futuro profesorado no conoce esta cualidad de la inteligencia, difícilmente llegará a conectar con sus alumnos de altas capacidades. En un principio se identificaron siete tipos diferentes de inteligencia: espacial, lingüística, lógico-matemática, corporal-kinestésica, musical, interpersonal e intrapersonal. Posteriormente (Sánchez \& Baena, 2017, p. 71) fueron ampliadas hasta nueve, con la inclusión de la inteligencia naturalista y espiritual, aunque existen estudios recientes que hablan de once tipos de inteligencias. A este respecto, destacan las aportaciones de Cotes y Gómez (2009), Gamboa, García y Beltrán (2013), Larivée (2010), Muñoz y Ayuso (2014), y Suárez, Maiz y Meza (2010). Otros autores como Tannenbaum (1991) o Renzulli y Reis (2016), enseñaron a aplicar esta conceptualización sobre inteligencia al trabajo educativo con las altas capacidades.

Según Higueras-Rodríguez y Fernández-Gálvez, "los sujetos capaces de altas realizaciones son aquellos con logro demostrado y/o capacidad potencial (ability) en cualquiera de las áreas siguientes: Capacidad intelectual general; aptitud académica específica; pensamiento creativo y productivo; artes visuales y representaciones; habilidad (ability) psicomotora; y habilidad de liderazgo" (2017, p. 151).

Según Agudo (2017), y Barrera et al. (2008), los niños con altas capacidades presentarían las siguientes cualidades a tener en cuenta por el profesorado que atienda al alumno y su familia: Dominio precoz y fluido del lenguaje oral, vocabulario amplio; interés y habilidad lectora temprana; comprensión de conceptos abstractos y complejos para su edad cronológica; establece relaciones y diferencias en los conocimientos; cuestionamiento de la realidad, habilidad creativa, capacidad para generar ideas nuevas a partir de información que conoce; capacidad de observación y escucha; gran capacidad memorística; utiliza con soltura e innovación materiales presentes en el entorno, expresión original en sus expresiones escritas, orales, etc.; facilidad en la resolución de problemas complejos, abordando su solución desde diferentes versiones; aburrimiento en tareas mecánicas, repetitivas; preferencia por la indagación o experimentación; finaliza con rapidez la tarea encomendada; se siente cómodo con personas de mayor edad, tiene confianza en sí mismo, es perfeccionista y constante; capacidad para simpatizar con otras personas, tiene muchas aficiones.

De igual modo, las necesidades educativas que suelen presentar la mayoría de los individuos en edad escolar con altas capacidades, según recogen Agudo (2017) y Barrera et al. (2008), y que deben ser atendidas, son: Entorno que fomente su actitud creativa u originalidad; flexibilización en los tiempos y ritmos de aprendizaje según intereses; facilitar dinámicas que potencien la autonomía, independencia: programación de tareas que le supongan nuevos retos; favorecer la integración en el grupo-clase, fomentando el sentimiento de pertinencia, estima y respeto entre compañeros; disponer de recursos educativos para mejorar la oferta ordinaria; reconocer su trabajo, y animar hacia nuevos logros; cultivar y ejercitar las habilidades sociales, aspectos de la personalidad, motivación; adaptaciones de currículo con tareas con mayor nivel de dificultad, amplitud, labores de investigación; evitar por completo tareas repetitivas; y la planificación de hábitos y técnicas de estudios ajustadas a su rendimiento y potencial (Agudo, 2017). 
Pero, el contexto educativo formal en muchas ocasiones no es capaz de resolver de forma óptima todas las necesidades educativas especiales que presentan los niños con altas capacidades. Estudios como los de Alencar (2008), Soriano (2008) constatan que el potencial superior de los niños con altas capacidades, en un ambiente educacional típico, no está atendido de forma adecuada a sus necesidades. Según Higueras-Rodríguez y Fernández-Gálvez de manera general, la enseñanza regular está dirigida hacia el alumnado medio y por debajo de la media, pero el alumnado con altas capacidades, además de ser dejado de lado en este sistema, es percibido con desconfianza por el profesorado que se sienten amenazado o cuestionado, así como presionado con sus preguntas y comentarios (2017, p. 153). Para Cañete (2010), los niños y niñas excepcionales por exceso han recibido poca atención y ayuda para el desarrollo óptimo de sus habilidades.

Es por todo ello, que autores como Cano (2002), o Higueras-Rodríguez y FernándezGálvez (2017), consideren que estos niños requieran de programas educativos diferenciados y servicios más allá de lo que suministran los programas escolares normales en orden a realizar sus contribuciones a sí mismos y a la sociedad. Según Tourón es preciso que se contemplen de modo más adecuado las diferencias de capacidad, intereses, motivaciones y competencias de los estudiantes, "convirtiendo las escuelas en ámbitos de desarrollo del talento, a través de un aprendizaje tan personalizado como sea posible". (2020, p. 29).

Autores como Sternberg y Davidson (2004), Sternberg (2004), Wallace y Erikson (2006), Pfeiffer (2011), Martínez, Mercé y Guirado (2012), o Torrego (2012), de una forma u otra, han defendido la importancia de tener una escuela preparada con un profesorado formado, para asegurar la calidad educativa de los menores con altas capacidades, potenciando la educación inclusiva (en sus diferentes formas), y evitar el tan temido fracaso escolar. Otro ejemplo de ello es el Dictamen del Comité Económico y Social Europeo (486/2013), titulada Liberar el potencial de los niños y los jóvenes con gran capacidad intelectual dentro de la Unión Europea, que establecía en su apartado 1.6, como uno de los elementos fundamentales para la atención educativa de los menores con altas capacidades, la formación inicial y permanente del profesorado acerca del perfil y las características del alumnado con altas capacidades, su detección y la atención educativa que necesitan. En la misma línea, el Consejo Superior de Expertos en Altas capacidades, junto con el Consejo General de Colegios Oficiales de Médicos de España, la Confederación Española de Asociaciones de Altas capacidades (CEAS), el Instituto Internacional de Altas capacidades, y la Fundación para la Formación (FFOMC), en 2014, crearon una guía científica sobre las altas capacidades, declarada de interés científico y profesional (Resolución de 27/01/2014), defendían la formación del profesorado para alumnos de altas capacidades, haciendo especial énfasis en el hecho diferencial de este perfil de alumnado, y por tanto, de una especial cualificación para una adaptación curricular muy específica, basada fundamentalmente en la educación inclusiva. Todo ello es conveniente realizarlo a través de la potenciación de la formación tanto en Grados, como en Másteres y Doctorados (Consejo Superior de Expertos en Altas capacidades, 2014).

A partir de estas reflexiones, se planteó la hipótesis de partida: El profesorado no cuenta con suficiente formación de grado y posgrado para poder trabajar en el aula las necesidades educativas especiales de los menores con Altas capacidades. 
Es este sentido, cuando el profesorado no posee una adecuada o suficiente formación, se genera un impacto negativo en el alumnado con altas capacidades. Tourón, Fernández y Reyero (2002) recalcan que la formación en altas capacidades no sólo es esencial para que el profesorado disponga de herramientas pedagógicas suficientes, sino también para ser capaz de empatizar con sus alumnos de Altas capacidades y poder dar respuesta a sus necesidades, o incluso en su papel crucial en la detección en el aula de sujetos con altas capacidades. Este impacto de la formación del profesorado ha sido abarcado por distintos estudios, como los realizados por Tallent-Runnels y Tirri (1998), en una comparativa entre Finlandia y Estados Unidos, o como el llevado a cabo en España por Fernández et al. (2009). En todos ellos se analizaba el impacto positivo y mejor desarrollo educativo de los menores con altas capacidades cuando el profesorado presenta una correcta formación y deja atrás determinados prejuicios y actitudes frente a las altas capacidades, y empieza a comprenderlas como necesidades educativas especiales. Tras el estudio llevado a cabo por Liesa et al. (2019) a 402 estudiantes de Grados de Magisterio, concluyeron que la falta de formación sobre altas capacidades de los egresados en Magisterio no les ofrecía la posibilidad de acceder a la práctica laboral como profesionales verdaderamente competentes. Para las autoras, esta falta de formación daña directamente a los niños y niñas superdotados, rompiendo por completo el concepto de escuela inclusiva.

Por otro lado, Mendioroz, Rivero, y Aguilera (2019), indicaron que la falta de formación del profesorado en altas capacidades, aumentaban sus limitaciones para ser capaces de dar respuestas a las necesidades educativas especiales de estos niños y niñas dentro del aula. Gómez, et al. (2019) nos indican que cuando el profesorado no tiene la suficiente formación sobre altas capacidades, sus alumnos se sienten incomprendidos, rechazados, y en muchos casos abocados a fracasar en etapas posteriores por su desmotivación y falta de estímulo. Por ello se hace tan necesaria una correcta formación del profesorado.

Tras lo expuesto, este estudio de investigación se propuso alcanzar el siguiente objetivo: Analizar el nivel de formación que el profesorado tiene a su disposición en temas de altas capacidades desde su formación en Grado y Posgrados en Andalucía.

\section{Método}

\section{Población y Muestra}

La muestra que se utilizó en este estudio, fueron las Universidades Públicas andaluzas (Universidad de Almería; Universidad de Cádiz; Universidad de Córdoba; Universidad de Granada; Universidad de Huelva; Universidad de Jaén; Universidad de Málaga; Universidad de Sevilla; Universidad Internacional de Andalucía; Universidad Pablo de Olavide) más la UNED, analizando en todas ellas sus grados y postgrados, vinculados a las plantillas de referencia de los Centros educativos de primaria de Andalucía: Grados en Educación Primaria; Grados de Educación Infantil; Grado de Pedagogía. Esto supuso la revisión de un total de 35 asignaturas de grado, y un total de 184 temas específicos o módulos específicos de contenido en referencia a Necesidades Educativas Especiales o Altas capacidades. En los posgrados, se revisaron 8 títulos que quedan recogidos en la Tabla 1 . 
Tabla 1

Posgrados analizados.

\begin{tabular}{|c|c|}
\hline Universidad & Título \\
\hline \multirow[t]{3}{*}{ Universidad de Sevilla } & $\begin{array}{l}\text { Necesidades Educativas Especiales y Atención a la } \\
\text { Diversidad en la Escuela }\end{array}$ \\
\hline & $\begin{array}{l}\text { Psicología de la Educación. Avances en Intervención } \\
\text { Psicoeducativa y Necesidades Educativas Especiales }\end{array}$ \\
\hline & Psicopedagogía \\
\hline $\begin{array}{l}\text { UNED (Universidad Nacional } \\
\text { de Educación a Distancia) }\end{array}$ & Tratamiento educativo de la diversidad \\
\hline Universidad de Huelva & Educación Especial \\
\hline Universidad de Córdoba & Educación Inclusiva \\
\hline Universidad de Almería & Educación Especial \\
\hline Universidad de Granada & Intervención Psicopedagógica \\
\hline
\end{tabular}

\section{Instrumento}

A través de técnicas de análisis documental, se realizó una recolección cuantitativa y pormenorizada de datos acerca de grados y posgrados relacionados con la educación, y en especial, ahondando en los contenidos que forman a los futuros profesionales en materia de altas capacidades. Para ello se midieron a través de análisis documental las siguientes 5 variables de formación de grado y posgrado en altas capacidades: Asignaturas obligatorias y optativas que presentaban algún contenido sobre altas capacidades en los grados y posgrados relacionados con la educación en Andalucía; proporción de créditos obligatorios y optativos donde se daba algún contenido sobre altas capacidades respecto al total de créditos en los grados relacionados con la educación en Andalucía; proporción de créditos obligatorios y optativos exclusivos de altas capacidades respecto al total de créditos en los grados relacionados con la educación en Andalucía; tiempo (en horas) empleado en contenidos de altas capacidades en base a créditos de contenido exclusivo de altas capacidades en los grados relacionados con la educación en Andalucía; proporción de créditos (porcentaje) exclusivos de altas capacidades respecto al total de créditos en los posgrados relacionados con la educación en Andalucía. Para facilitar la recolección de datos, se generó una hoja rejilla (con soporte en Excel), a modo de rúbrica, que fue utilizada por el equipo investigador.

\section{Procedimiento de recogida y análisis de datos}

Teniendo en cuenta el objetivo e hipótesis, se llevó a cabo un análisis de los planes de estudios universitarios de grado y posgrado vinculados a las altas capacidades en las universidades públicas de Andalucía. El estudio se realizó entre 2019-2020. La investigación estuvo sujeta al Código de Ética de Trabajo Social y de la Federación 
Internacional de Trabajadores Sociales, y a la Ley Orgánica 3/2018, de 5 de diciembre, de Protección de Datos Personales y garantía de los derechos digitales.

Nuestra investigación desarrolló el siguiente procedimiento:

Primero, selección de la muestra y acceso a las bases de datos oficiales, a través de los programas y planes de estudio de todos los ciclos de grado y posgrado analizados en todas las universidades seleccionadas.

Segundo, recopilación de los datos utilizando las variables del estudio.

Tercero, creación de diferentes indicadores estadísticos que permitieron la comparación de resultados, variables y de todas las universidades y ciclos formativos estudiados.

Cuarto, análisis estadístico y tratamiento de los datos a través de Excel, y programa informático IBM SPSS Statistics 25.

\section{Resultados}

La primera de las variables analizadas fue el número de asignaturas obligatorias y optativas que presentan algún contenido sobre altas capacidades en los Grados relacionados con la Educación en Andalucía (ver tabla 2).

Tabla 2

Asignaturas obligatorias y optativas sobre altas capacidades

\begin{tabular}{lllr}
\hline & $\begin{array}{c}\text { Asignaturas } \\
\text { Optativas }\end{array}$ & $\begin{array}{c}\text { Asignaturas } \\
\text { Obligatorias }\end{array}$ & $\begin{array}{l}\text { \% sobre el total } \\
\text { de formación del } \\
\text { Grado }\end{array}$ \\
\hline Educación Primaria (Univ. Almería) & 0 & 1 & 2,5 \\
Educación Primaria (Univ. Cádiz) & 2 & 0 & 5 \\
Educación Infantil (Univ. Córdoba) & 0 & 1 & 2,5 \\
Educación Primaria (Univ. Córdoba) & 2 & 0 & 5 \\
Educación Primaria (Univ. Granada) & 0 & 1 & 2,5 \\
Educación Primaria (Univ. Huelva) & 1 & 1 & 5 \\
Educación Infantil (Univ. Jaén) & 3 & 2 & 12,5 \\
Educación Primaria (Univ. Jaén) & 2 & 1 & 7,5 \\
Pedagogía (Univ. Málaga) & 1 & 0 & 2,5 \\
Educación Primaria (Univ. Málaga) & 0 & 1 & 2,5 \\
Pedagogía (Univ. Sevilla) & 1 & 1 & 5 \\
Educación Primaria (Univ. Sevilla) & 2 & 1 & 7,5 \\
Pedagogía (UNED) & 1 & 1 & 5 \\
\hline
\end{tabular}

Se puede observar que la carga de asignaturas optativas sobre altas capacidades fue mayor que las asignaturas obligatorias. Pero en general resultaron realmente bajas ambas; hubo titulaciones que no tenían ninguna asignatura obligatoria con estos con- 
tenidos, y la que más tuvo fue la Universidad de Jaén con 2 asignaturas obligatorias en el Grado de Educación Infantil. Si atendemos a la figura 1, se puede ver que los porcentajes de créditos obligatorios de las asignaturas que tuvieron alguna presencialidad de altas capacidades no superaron en ningún caso el 5\% de un grado, y la media total fue gracias a la Universidad de Jaén, que fue la que más destacó en concienciación para este colectivo en cuanto a presencialidad de contenidos en varias asignaturas. Sin la Universidad de Jaén, los porcentajes no llegaban a superar un $2.50 \%$, valor que se acercaba más a la media total de créditos obligatorios relacionados con este colectivo en las universidades andaluzas, que es de un $2.12 \%$. Haciendo lo mismo para los créditos optativos, se observó que los valores iban desde $0 \%$ hasta $7.50 \%$ (aunque este valor máximo fue único en Educación Infantil de Jaén), pasando por valores más frecuentes como $2.50 \%$ y $5 \%$. Así, la media total de créditos optativos en las universidades andaluzas fue de $3.08 \%$. Recalcamos de nuevo que estos créditos son de las asignaturas en su totalidad, siendo el contenido de altas capacidades contemplado, pero no necesariamente exclusivo y profundizado.

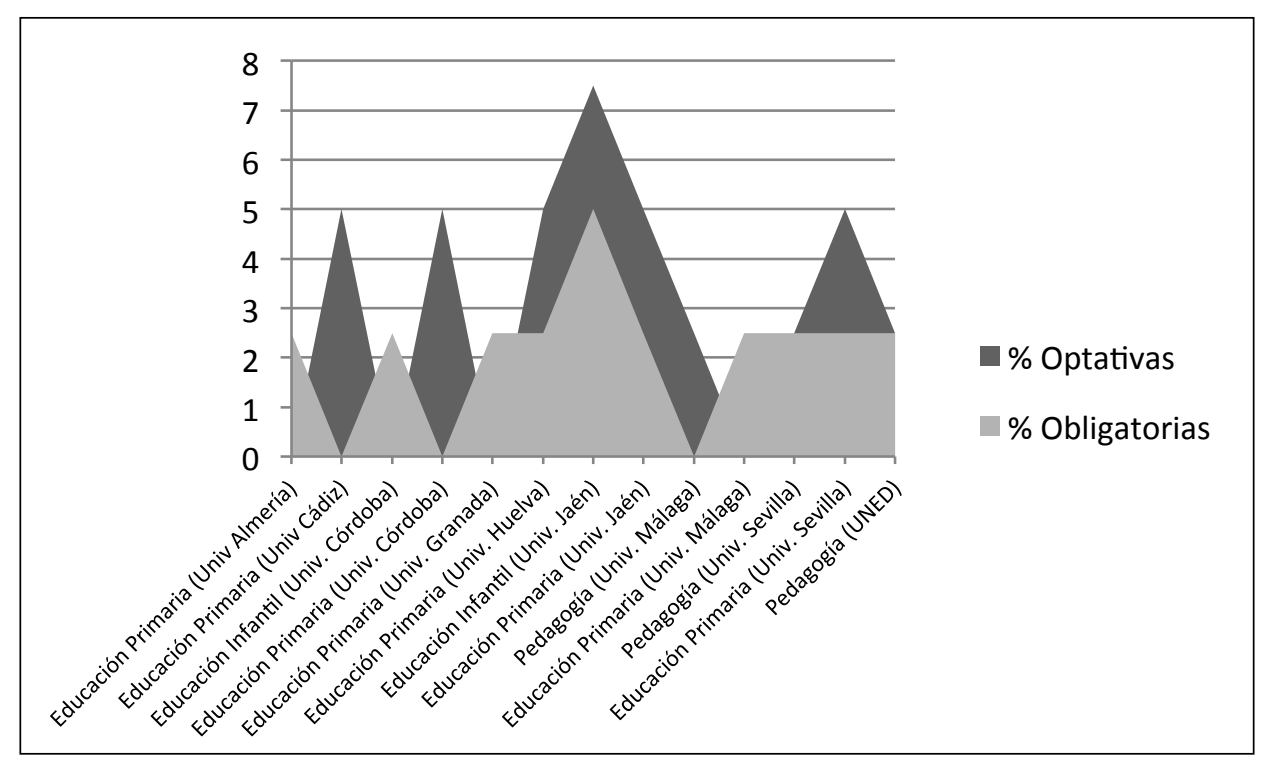

Figura 1. Porcentaje de optatividad y obligatoriedad

Para observar mejor la información, se realizó una comparativa entre el número de asignaturas con contenido en altas capacidades, y el total de asignaturas del título, para hallar el peso en porcentaje sobre el total de formación del Grado (ver tabla 2). La media en el porcentaje del peso de las asignaturas en altas capacidades en relación al total de asignaturas del título, se queda en el $5 \%$, un porcentaje muy bajo: Aquí encontramos titulaciones (5) con un peso de $2.5 \%$, destacando algo más la Universidad de Jaén con un peso del $12.5 \%$.

Recordemos que los títulos de Grado del Espacio Europeo de Educación Superior se corresponden con 240 créditos ECTS (sin contar varias excepciones en titulaciones 
que no afectan a este análisis), lo cual supone que en cada curso académico se realizan 60 créditos. Un crédito académico equivaldría a 25 horas, donde se incluyen las horas académicas con acompañamiento docente y el resto de tiempo que se invierte en actividades autónomas de estudio, prácticas o preparación de exámenes. Esto quiere decir que, por ejemplo, los grados donde sólo existe una asignatura obligatoria relacionada con altas capacidades, se imparten un curso de 150 horas, una temporalización discutible si se compara con las 6.000 horas restantes que conforman el grado. Para concretar más, también se analizará qué parte de esas asignaturas se corresponde realmente con enseñanza relacionada con altas capacidades, puesto que esas 150 horas de cada asignatura, frecuentemente, se reparten entre diversos contenidos al margen de altas capacidades.

Por esta razón, se procedió a construir un indicador que permitiera apreciar el peso real del contenido exclusivo de altas capacidades en cada asignatura y en cada grado, sean uno o varios epígrafes, uno o varios temas o toda la asignatura. Así, este indicador (porcentaje) está conformado de la siguiente forma:

$\underline{\text { Cantidad de créditos relacionados con AACC }} \times 100$ Cantidad total de créditos

Se utilizaron créditos ECTS como unidad, lo que nos permitió realizar análisis comparativo en cualquier universidad. Concretando, la cantidad total de créditos, para la selección de asignaturas, fue siempre de 6. En cuanto a la cantidad de créditos relacionados con altas capacidades, estos se obtuvieron de la división que tenía como numerador el total de créditos de la asignatura (6 para nuestros casos) y como denominador el número de temas que tenía dicha asignatura, resultado que se multiplicó por el número de temas relacionados con altas capacidades.

En esa línea, el resultado fue el peso relativo que tenían los temas que incluyen altas capacidades en créditos, pero para ver exactamente esto, el valor de número de temas relacionados con altas capacidades se correspondió con los epígrafes/temas específicos que incluían este colectivo. Es decir, si el contenido señalado no alcanzaba 1 tema o no era un número entero de temas, se expresó su peso en decimales, teniendo en cuenta los epígrafes específicos para altas capacidades. La fórmula para hallar estos créditos relacionados fue:

Cantidad de créditos relacionados AACC por asignatura $=\frac{\text { Cantidad total de créditos }}{\text { Número de temas }} \times$ Número de temas relacionados con AA

Tras aplicar este indicador a cada asignatura se hizo una agrupación en asignaturas obligatorias y asignaturas optativas respectivamente, a través de sumatorios, y a su vez, se agruparon por titulación y universidad, comparando los totales de porcentaje obligatorio y porcentaje optativo con los créditos totales del grado (240 créditos). El resultado se expresa a continuación (ver tabla 3). 
Tabla 3

Proporción de créditos obligatorios y optativos exclusivos de altas capacidades respecto al total de créditos en los grados relacionados con la educación en Andalucía (porcentaje).

\begin{tabular}{llll}
\hline & \multicolumn{1}{c}{$\begin{array}{c}\text { Índice de optatividad } \\
(\%)\end{array}$} & \multicolumn{1}{c}{$\begin{array}{c}\text { Índice de obligatoriedad } \\
(\%)\end{array}$} \\
\hline Educación Primaria (Univ. Almería) & 0 & 0,13 \\
Educación Primaria (Univ. Cádiz) & 0,43 & 0 \\
Educación Infantil (Univ. Córdoba) & 0 & 0,42 \\
Educación Primaria (Univ. Córdoba) & 0,35 & 0 \\
Educación Primaria (Univ. Granada) & 0 & 0,1 \\
Educación Primaria (Univ. Huelva) & 0,23 & 0,28 \\
Educación Infantil (Univ. Jaén) & 0,95 & 0,61 \\
Educación Primaria (Univ. Jaén) & 0,61 & 0,25 \\
Pedagogía (Univ. Málaga) & 0,28 & 0 \\
Educación Primaria (Univ. Málaga) & 0 & 0,08 \\
Pedagogía (Univ. Sevilla) & 0,21 & 0 \\
Educación Primaria (Univ. Sevilla) & 0,1 & 0 \\
Pedagogía (UNED) & 2,5 & 0,31 \\
Media andaluza & $\mathbf{0 , 6 3}$ & $\mathbf{0 , 2 2}$ \\
\hline
\end{tabular}

En la tabla 3, se observa la proporción de créditos obligatorios y optativos exclusivos de altas capacidades respecto al total de créditos en los grados relacionados con la educación en Andalucía (porcentaje). Podemos comentarlo según cada universidad estudiada.

Por un lado encontramos a la Universidad de Almería con el Grado de Educación Primaria, con una asignatura básica con 1 tema (entre otros 4) donde 1/4 fue para este colectivo, y ninguna optativa. Esto resultaba en $0 \%$ de créditos optativos y $0.13 \%$ de créditos obligatorios a lo largo del grado.

En la Universidad de Cádiz se encuentra el Grado de Educación Primaria con dos optativas donde una parte de un tema respectivamente se asociaba con diferentes ritmos de aprendizaje y grados de capacidad, pero a groso modo. Así, el indicador es de $0.43 \%$ de créditos optativos y $0 \%$ de créditos obligatorios para altas capacidades.

En la Universidad de Córdoba estaba el Grado en Educación Primaria con sólo dos optativas que tocaban ligeramente las altas capacidades y ninguna asignatura obligatoria, con un $0.35 \%$ de créditos optativos y $0 \%$ de créditos obligatorios. Así también, se encontraba en esta misma universidad el Grado de Educación Infantil, con un tema dentro de una asignatura obligatoria que formaba sobre el colectivo objeto de estudio, representándose en el $0.42 \%$ de créditos obligatorios para altas capacidades, y al no existir asignaturas optativas, el valor de créditos opcionales en altas capacidades es de $0 \%$. 
En la Universidad de Granada, el Grado de Educación Primaria no poseía ningún tema específico dentro de una asignatura básica de necesidades educativas, por lo que se daba un $0.10 \%$ de créditos obligatorios, así como un $0 \%$ de créditos optativos al no poseer ninguna optativa que mantenga relación.

En la Universidad de Huelva, estaba el Grado de Educación Primaria con una asignatura básica con un tema de alta capacidad y una optativa donde la alta capacidad representaba un epígrafe dentro de un tema, por lo que se obtenía un $0.28 \%$ de créditos obligatorios y $0 \%$ de créditos optativos.

En la Universidad de Jaén se daba la mayor cantidad de contenidos relacionados con altas capacidades a nivel de asignaturas que la incluían, aunque como se ha comentado, el contenido de las asignaturas se repartía para varias necesidades educativas específicas. De esta forma, se encontraba, por un lado, el Grado de Educación Infantil con una asignatura básica de educación inclusiva donde un tema (entre otros 12) era de altas capacidades, y otra asignatura básica con un tema de desarrollo y aprendizaje del alumnado con altas capacidades (junto a otros 5). Así también, se daban tres asignaturas optativas asociadas. Una primera poseía un tema de altas capacidades (entre otros 14 temas). La segunda tenía un tema de intervención con altas capacidades (de un total de 5 temas inclusive), incorporando evaluación, identificación, problemática e intervención. Finalmente, la tercera optativa incluía un tema que unía las TIC y el alumnado con altas capacidades (además de otros 5 temas). Por otro lado, estaba el grado de Educación Primaria en la misma universidad con una asignatura obligatoria donde un tema (entre otros 9) era de altas capacidades, una optativa donde un epígrafe dentro de un tema, y otro tema, son específicos para altas capacidades, y otra optativa en la cual 1 tema de los 6 totales era para este colectivo. A pesar de tener mayor presencialidad de altas capacidades en diferentes asignaturas, el peso efectivo de contenidos era más bajo que en la UNED, donde se daban menos asignaturas, pero con más contenido dedicado a altas capacidades. Con todo lo anterior, el Grado de Educación Infantil poseía un $0.61 \%$ de créditos obligatorios y $0.95 \%$ de créditos optativos, mientras que el Grado de Educación Primaria se daba un $0.25 \%$ de créditos obligatorios y $0.61 \%$ de créditos optativos, destacando en ambas titulaciones por encima del resto de las universidades presenciales andaluzas.

La Universidad de Málaga poseía el Grado de Educación Primaria, la cual tenía una asignatura obligatoria con un epígrafe de altas capacidades, y ninguna optativa, resultando un $0.08 \%$ de créditos obligatorios con respecto al grado y $0 \%$ de créditos optativos. Por su parte, el Grado de Pedagogía en la misma universidad, sólo contaba con una asignatura optativa que incluía epígrafes relacionados con necesidades específicas como la alta capacidad, así como un tema específico para este colectivo, de forma que tenía un $0.28 \%$ de créditos optativos para altas capacidades y $0 \%$ de créditos obligatorios.

En el grado de Educación Primaria en la Universidad de Sevilla la asignatura obligatoria enfocaba las necesidades de forma general, por lo que las altas capacidades no se mencionaban en ningún epígrafe. Esto arroja un $0 \%$ de créditos obligatorios con el grado completo como referencia. Igualmente ocurría en las 2 asignaturas optativas, ofreciéndose conocimientos y herramientas para identificar necesidades en general, 
pero donde las altas capacidades no llegaban a ser un tema específico, por lo que se obtuvo un $0.1 \%$ de créditos optativos. En el Grado de Pedagogía para la misma universidad, se analizó lo respectivo en formas de educación inclusiva y diversidad a modo general, así como modelos, estrategias y centros para una educación sin segregación. En este caso, la asignatura más específica para altas capacidades era optativa, aunque el porcentaje de créditos de esta asignatura para este colectivo con respecto al total del grado fue de $0.21 \%$. De nuevo se obtuvo un $0 \%$ de créditos obligatorios.

En la UNED se encontraba el Grado de Pedagogía con una asignatura obligatoria donde un tema entre ocho estaba relacionado de forma concreta con altas capacidades. Otra asignatura era optativa, y toda ella se centraba exclusivamente en comprender y educar al alumnado en altas capacidades, así como estrategias para abordar el aprendizaje dentro de este marco. Por esto, aunque la Universidad de Jaén es la que más presencialidad de altas capacidades tenía en diferentes asignaturas, era la UNED la que obtuvo un mayor porcentaje real de créditos empleados para estos menores, con un $2.81 \%$ total que se reparte entre $2.50 \%$ optativo y $0.31 \%$ obligatorio.

Finalmente, la media andaluza dio como resultado un $0.63 \%$ de créditos optativos y $0.22 \%$ de créditos obligatorios en materia de altas capacidades. Esta fue la escasa formación que los futuros profesores y pedagogos tuvieron sobre altas capacidades.

A partir de aquí se realizó una comparativa entre proporción de créditos de asignaturas que incluían altas capacidades, con proporción de contenido exclusivo de altas capacidades, ambas respecto al total de créditos en los grados relacionados con la educación en Andalucía (porcentaje). Pudimos ver que la media en créditos obligatorios bajó de $2.12 \%$ a $0.22 \%$, y la media en créditos optativos hizo lo respectivo de $3.08 \%$ a $0.63 \%$. Además, la universidad de Jaén, que destacaba en el gráfico de presencialidad en diferentes asignaturas, quedaba relegada cuando se apreciaba el peso real de contenido en altas capacidades, mientras que ocurría lo contrario con la UNED. En general, todos los grados bajaron su porcentaje al especificar el número de créditos que eran exclusivos para altas capacidades.

Para comprender mejor estos resultados, y siendo la unidad elegida (créditos ECTS) traspasable a tiempo que se emplea en formación, se procedió a estudiar cuánto tiempo de estas titulaciones a lo largo de todo el grado se invierte en materia de altas capacidades. Los resultados se pueden apreciar en la tabla 4.

Tabla 4

Tiempo (horas) empleado en contenidos de altas capacidades en base a créditos de contenido exclusivo de altas capacidades en los grados relacionados con la educación en Andalucía (\%).

\begin{tabular}{lrr}
\hline & $\begin{array}{c}\text { Horas optativas en } \\
\text { AACC }\end{array}$ & $\begin{array}{c}\text { Horas obligatorias en } \\
\text { AACC }\end{array}$ \\
\hline Educación Primaria (Univ. Almería) & 0 & 7,5 \\
Educación Primaria (Univ. Cádiz) & 25,72 & 0 \\
Educación Infantil (Univ. Córdoba) & 0 & 25 \\
\hline
\end{tabular}




\begin{tabular}{lrr}
\hline & $\begin{array}{c}\text { Horas optativas en } \\
\text { AACC }\end{array}$ & $\begin{array}{c}\text { Horas obligatorias en } \\
\text { AACC }\end{array}$ \\
\hline Educación Primaria (Univ. Córdoba) & 21,25 & 0 \\
Educación Primaria (Univ. Granada) & 0 & 6,25 \\
Educación Primaria (Univ. Huelva) & 13,5 & 16,67 \\
Educación Infantil (Univ. Jaén) & 56,95 & 36,54 \\
Educación Primaria (Univ. Jaén) & 36,67 & 15 \\
Pedagogía (Univ. Málaga) & 16,67 & 0 \\
Educación Primaria (Univ. Málaga) & 0 & 5 \\
Pedagogía (Univ. Sevilla) & 12,5 & 0 \\
Educación Primaria (Univ. Sevilla) & 6,25 & 0 \\
Pedagogía (UNED) & 150 & 18,75 \\
Media andaluza & 37,72 & $\mathbf{1 3 , 0 7}$ \\
\hline
\end{tabular}

Se puede observar que durante los 4 años que posee un grado, las asignaturas obligatorias empleaban una media de 13'07 (13 horas con 4'2 minutos, exactamente) mientras que para las asignaturas optativas el valor total posible (teniendo en cuenta que no tenían por qué cursarse) ascendía a 37,72 horas (37 horas con 43'2 minutos). Profundizando un poco más en estos datos, los valores en las titulaciones seleccionadas rondaban valores entre 0 y 25-30 horas invertidas en altas capacidades durante todo el grado. Destacaba la UNED, por ser la que más invertía en esto, con 150 horas optativas, aunque con 18 horas con 45 minutos obligatorios, y la Universidad de Jaén, por poseer las dos titulaciones con mayor tiempo invertido de entre las universidades presenciales. Aun así, en cuanto al resto, no dejaban de ser valores relativamente bajos comparados con el tiempo total del grado, que es de 6.000 horas.

Por otro lado, se analizaron los posgrados, y más en concreto la proporción de créditos exclusivos de altas capacidades respecto al total de créditos en los posgrados relacionados con la educación en Andalucía. Se observó por ejemplo que en la Universidad de Sevilla se ofrecía este tipo de formaciones de 1 año de duración y 60 créditos. En concreto, existían tres. Un primero estaba encaminado a Necesidades Educativas Especiales y Atención a la Diversidad en la Escuela, con $0 \%$ de contenido en altas capacidades. El segundo se denominaba Psicología de la Educación. Avances en Intervención Psicoeducativa y Necesidades Educativas Especiales, de nuevo con $0 \%$ en altas capacidades. Y finalmente un tercer máster en Psicopedagogía, también con $0 \%$ en cuanto al contenido de altas capacidades.

Por su parte, la UNED ofrecía un máster de 2 años en Tratamiento Educativo de la Diversidad que poseía 5 créditos (una asignatura obligatoria completa) relacionados con altas capacidades de los 90 totales, es decir, un 4.17\% del máster. La Universidad de Huelva otro similar, de Educación especial, aunque de 1 año de duración, que poseía un $1.67 \%$ de contenido en este colectivo, en base a $1 / 3$ de una asignatura obligatoria de 3 créditos de los 60 créditos totales. La Universidad de Córdoba existía otro máster de 
Educación inclusiva, también de 1 año, que fomentaba la igualdad de oportunidades, aunque con $0 \%$ de contenido para altas capacidades. La Universidad de Granada ofrecía un posgrado de Intervención psicopedagógica, con nuevamente un 1 de duración y $0 \%$ en el colectivo señalado. Por último, la Universidad de Almería poseía un máster en Educación especial, al igual que la Universidad de Huelva, aunque en este caso con un $5 \%$ posible de contenido en altas capacidades respecto al total del posgrado gracias a una asignatura optativa específica.

En cualquier caso, los másteres mencionados no se centraban exclusivamente en altas capacidades, siendo la media de créditos empleados para este colectivo de $1.35 \%$ respecto al total del posgrado.

\section{Discusión y Conclusiones}

Este estudio pudo cumplir su objetivo: Analizar el nivel de formación que el profesorado tiene a su disposición en temas de altas capacidades desde su formación en Grado y Posgrados en Andalucía. En este sentido fue destacable que en los grados relacionados con la educación de las universidades públicas andaluzas, las asignaturas relacionadas con diversidad son escasas, y aún más las que ahondan el tema de menores con altas capacidades. Los datos arrojados muestran que las asignaturas obligatorias que forman a los estudiantes acerca de esta situación específica no eran más de 2 en todo el recorrido académico y, en algunos casos, incluso no había asignaturas obligatorias. En cuanto a las optativas, los números eran parecidos, aumentando un poco, pero al no ser obligatorias, el alumnado no tiene porqué formarse en estas materias (o no matricularse en todas) a menos que lo decida por propia voluntad, de forma que es posible que profesionales de educación se vean sin conocimientos ni herramientas para este colectivo si escogieron asignaturas optativas destinadas a otras cuestiones. Tal y como se ha desarrollado en la descripción de resultados, hay una ausencia de formación importante para los futuros egresados en grados de educación sobre las altas capacidades, tanto en términos de créditos obligatorios $\left(0^{\prime} 22 \%\right.$ de créditos obligatorios como media andaluza) como en los créditos optativos ( $0^{\prime} 63 \%$ de media andaluza). Añadido a esto, las asignaturas mencionadas se compartían con otros contenidos, por lo que en última instancia el tiempo empleado en altas capacidades es ínfimo (llegando a ser un tema o incluso un único epígrafe en toda la titulación). La mayor o menor adecuación en el trato a los menores con altas capacidades depende, por tanto, únicamente de que el estudiantado de grado tenga mayor afinidad por esta situación y decida enfocar sus opciones de elección a la misma. Esto deja a la escuela en condiciones de desventaja para los alumnos y alumnas de altas capacidades, pues no pueden ver atendidas sus necesidades educativas especiales en el sentido que exponían Agudo (2017) y Barrera, et al. (2008), por causa de falta de formación del personal del centro educativo. Esto constata que el contexto educativo formal en muchas ocasiones no es capaz de resolver de forma óptima todas las necesidades educativas especiales que presentan los niños con altas capacidades. Coincidimos pues con los estudios de Alencar (2008) y Soriano (2008).

En Andalucía, para buscar un profesional con un Grado de Universidad, con algo más de formación en Altas capacidades, hemos de hacerlo en la universidad no presencial, en la UNED, que es la que arroja mejores datos. 
Por otro lado, los másteres no terminan de tener una formación especializada y acaban siendo extensiones de los grados, por lo que aún no existen posgrados que ofrezcan formación exclusiva para el colectivo señalado. Únicamente 3 de los 8 posgrados seleccionados presentaban alguna asignatura relacionada con altas capacidades, y por lo general eran optativas. Así, en los posgrados se daban más irregularidades que en los grados, aunque cuando había el contenido requerido, solía ser una asignatura completa o gran parte de ella.

Por lo general, tanto los contenidos de grado como de posgrado apuntan a un déficit de los futuros egresados sobre altas capacidades (déficits, desigualdades o dificultades), dejando fuera la posibilidad de desarrollar potencialidades o formas de educación inclusiva, o diferenciada. Esto pone de manifiesto las reflexiones de Higueras-Rodríguez y Fernández-Gálvez (2017), sobre que la enseñanza regular está dirigida hacia el alumnado medio y por debajo de la media, donde el alumnado con altas capacidades, queda a un lado por el sistema, y en gran medida debido a la falta de formación del profesorado.

La formación externa es posible, en instituciones como por ejemplo: Fundación Avanza, en colaboración con Centro CADIS de niños y niñas con altas capacidades intelectuales, superdotados y talentos, con un curso totalmente especializado de 100 horas y homologado por el Ministerio de Educación, Cultura y Deporte (CADIS, 2020); UNIR (2020) (universidad privada reconocida por la Comunidad Autónoma de La Rioja y el Espacio Europeo de Educación Superior) ofrece un curso online de Experto Universitario en Altas capacidades y Desarrollo del Talento que se enfoca en la naturaleza y las características de los menores con altas capacidades (nivel cognitivo, afectivo, evolutivo y socio-emocional); la Asociación para la formación el ocio y el empleo AFOE (2020), que posee un curso de Atención educativa para el alumnado con altas capacidades homologado y acreditado por la Universidad Rey Juan Carlos y Universidad de Nebrija (APPF, 2020) de 100 horas; la propia Universidad Rey Juan Carlos ofrece otro curso denominado 'Las altas capacidades intelectuales en el aula', aunque de menor duración (19 horas); o la Fundación el Mundo del Superdotado (2020) con un curso de Superdotación y Altas capacidades para la formación del profesorado o para padres de menores con superdotación, homologado por el Ministerio de Educación, Cultura y Deporte. Pero al igual que ocurre con las asignaturas optativas, matricularse depende de la voluntad propia del profesional. Esto supone un problema para los menores escolarizados con altas capacidades, y tal y como lo recogen Sternberg y Davidson (2004), Sternberg (2004), Wallace y Erikson (2006), Pfeiffer (2011), Martínez, Mercé y Guirado (2012), o Torrego (2012), hay que buscar una escuela preparada con un profesorado formado, para asegurar la calidad educativa de los menores con altas capacidades, potenciando la educación inclusiva (en sus diferentes formas), y evitar el tan temido fracaso escolar.

A modo de conclusión, verificamos nuestra hipótesis de partida: El profesorado no cuenta con suficiente formación de grado y posgrado para poder trabajar en el aula las necesidades educativas especiales de los menores con Altas capacidades.

Por tanto se hace necesaria una expansión de la formación del profesorado, tal y como lo recogen el Dictamen del Comité Económico y Social Europeo (486/2013), y el Consejo Superior de Expertos en Altas capacidades, junto con el Consejo General 
de Colegios Oficiales de Médicos de España, la Confederación Española de Asociaciones de Altas capacidades (CEAS), el Instituto Internacional de Altas capacidades, y la Fundación para la Formación (FFOMC), (Consejo Superior de Expertos en Altas capacidades, 2014), especialmente en los Grados, Másteres y Programas de Doctorado. Todo ello para asegurar que la escuela desarrolle de modo más adecuado las diferencias de capacidad, intereses, motivaciones y competencias de los estudiantes, donde se potencie el talento, a través de un aprendizaje tan personalizado como sea posible. (Tourón, 2020). En este sentido, y apoyándonos en argumentos desarrollados por Tourón, et al. (2002), Tallent-Runnels y Tirri (1998), Fernandez et al. (2009), Liesa et al. (2019), Mendioroz et al. (2019) o Gómez et al (2019), una correcta formación del profesorado evitará un impacto negativo en los niños y niñas con altas capacidades, asegurando una conexión con la escuela inclusiva, donde se prevenga el fracaso escolar, las frustraciones, la desmotivación, y el rechazo del colectivo.

Como propuesta de formación al profesorado, estos deberían trabajar contenidos de altas capacidades sobre: Características generales y teorías más relevantes sobre altas capacidades; detección y evaluación en altas capacidades; intervención educativa en las altas capacidades; programas de enriquecimiento tanto en el ámbito escolar como extraescolar; la creatividad y la altas capacidades; el papel del docente en el aula con altas capacidades; las estrategias de aprendizaje para el alumnado con altas capacidades; dificultades asociadas a las altas capacidades; y por supuesto, familia, escuela y altas capacidades. Con ello, se podrían atender las necesidades educativas que suelen presentar la mayoría de los individuos en edad escolar con altas capacidades, según recogen Agudo (2017) y Barrera et al. (2008).

\section{Referencias}

AFOE (2020). Atención educativa para el alumnado con altas capacidades. Recuperado el 07/04/2020 de: https://www.afoe.org/curso/atencion-educativa-alumnado-altascapacidades/

Agudo, N. (2017). A student with high abilities in my classroom, Now what? Revista Nacional e Internacional de Educación Inclusiva, 10(1), 265-277. Recuperado de: http:// www.revistaeducacioninclusiva.es/index.php/REI/article/view/292/287

Alencar, E. (2008). Social-emotional difficulties of the student with high abilities. Revista de psicología, 26(1), 43-62. Recuperado de: http://revistas.pucp.edu.pe/index.php/ psicologia/article/view/1123

APPF (2020). Las altas capacidades en el aula. Recuperado el 1/04/2020 de: https://www. appf.edu.es/catalogo-de-cursos/las-altas-capacidades-intelectuales-en-el-aula/

Barrera, A., Durán, R., González, J., \& Reina, C. (2008). Handbook for students with specific educational support needs due to their high intellectual capacity. Sevilla: Junta de Andalucía.

CADIS. (2020). Curso AACC 2019. Recuperado el 28/03/2020 de: http://www.centrocadis. com/formacion/

Cano, I. (2002). Alumnado con altas capacidades intelectuales: Propuesta de intervención. La Rioja: Universidad internacional de la Rioja. 
Cañete, M.M. (2010). La educación en niños y niñas superdotados. Revista digital de innovación y experiencias educativas, 30, 1-10. Recuperado de: https://cutt.ly/PtHWtCr

Coleman, L.J., \& Cross, T. (2005). Definitions and models of giftedness. In Being gifted in school, 2nd ed. (pp. 1-25). Waco, TX: Prufrock Press.

Consejo Superior de Expertos en Altas capacidades, (2014). Guía científica de las altas capacidades. España: Consejo Superior de Expertos en Altas capacidades. Recuperado el 07/07/2020 de http://altascapacidadescse.org/shop/index.php

Cotes, S., \& Gómez, M. (2009). Theory of Multiple Intelligences: A Contribution to Higher Education. Academia Libre (7), 29-35. Recuperado de: https://cutt.ly/ItHESDb

Dictamen del Comité Económico y Social Europeo (486/2013/UE), de 19 de Enero de 2013, relativa a Liberar el potencial de los niños y los jóvenes con gran capacidad intelectual dentro de la Unión Europea (DOUE 2013/C 76/01).

Curso de Superdotación y Altas capacidades para la formación del profesorado. Recuperado el 28/02/2020 de: https://cutt.ly/StHEmZY

Fernandez, M. D., Álvarez, B., Ocio, E. S., Rayo, J., \& Se-púlveda, F. (2009). Diagnóstico y educación de los más capaces. Madrid: Editorial UNED.

Gamboa, M., García, Y., \& Beltrán, M. (2013). Pedagogical and didactic strategies for the development of multiple intelligences and autonomous learning. Revista de Investigaciones UNAD, 12(1), 101-128. Recuperado de: https://cutt.ly/OtHETmQ

Gardner, H. (1998). A Reply to Perry D. Klein's 'Multiplying the problems of intelligence by eight". Canadian Journal of Education 23 (1), 96-102. doi:10.2307/1585968. JSTOR 1585790

Gómez, C., Gonzalo, M., de León, B., \& Mendo, S. (2019). Extremadura, verde, blanca, negra. Avances en altas capacidades. Revista INFAD de Psicología. International Journal of Developmental and Educational Psychology., 3(1), 65-74. doi:https://doi.org/10.17060/ ijodaep.2019.n1.v3.1451

Higueras-Rodríguez, L., \& Fernández-Gálvez, J. (2017). The role of the family in the education of children with high intellectual capacities. International Journal of Educational Research and Innovation (IJERI), 7, 149-163. Recuperado de: https:/cutt.ly/0tHEPBS

Larivée, S. (2010). Gardner's Multiple Intelligences: Discovery of the Century or Simple Political Righteousness? Revista Mexicana de Investigación en Psicología, 2(2), 115-126. Recuperado de: https://cutt.ly/qtHEU5G

Liesa, M., Latorre, C. y Vázquez, S. (2019) La formación inicial de los Graduados de Magisterio en la atención al alumnado con altas capacidades: retos y oportunidades para la educación inclusiva. Profesorado. Revista de Curriculum y Formación del Profesorado 23(4), 46-66. DOI: 10.30827/profesorado.v23i4.11428

Martínez, M. y Guirado, Á. (2012). Altas capacidades intelectuales. Pautas de actuación, orientación, intervención y evaluación en el período escolar. Barcelona: Editorial Graó.

Mendioroz, A. M., Rivero, P. y Aguilera, P. (2019). Una propuesta de formación docente para responder a las altas capacidades en la escuela inclusiva. Profesorado. Revista de Curriculum y Formación del Profesorado 23(1), 265-284. DOI:10.30827/profesorado. v23i1.9154

Muñoz, M., \& Ayuso, M. (2014). Multiple Intelligences, Eight Different Ways to Learn? Escuela abierta: revista de Investigación Educativa (17), 103-116. Recuperado de: https:// dialnet.unirioja.es/descarga/articulo/4801385.pdf 
Pfeiffer, S. I. (2011). Current Perspectives on the Identification and Assessment of Gifted Students. Journal of Psychoeducational Assessment, 30(1), 3-9. DOI: https://doi. org/10.1177/0734282911428192

Renzulli, J. S. (2016). The three-ring conception of giftedness. In S. M. Reis (Ed.). Reflections On Gifted Education (pp. 55 - 86). Waco, TX: Prufrock Press.

Renzulli, J. S., \& Reis, S. M. (2016). Reflections on gifted education: Critical works. In S. Renzulli and Colleagues. Waco, TX: Prufrock Press.

Sánchez, A., \& Baena, M. (2017). Identificación del alumno con altas capacidades intelectuales: ¿responsabilidad del maestro o del departamento de orientación educativa y psicopedagógica? Aula de Encuentro, 1(19), 69-91. Recuperado de: https:// revistaselectronicas.ujaen.es/index.php/ADE/article/view/3313

Soriano, E. (2008). Social-emotional difficulties of students with high skills. Revista de Psicología, 26(1), 43-62. Recuperado de: https://cutt.ly/ytHEJEo

Sternberg, R J. (2004) Definitions and conceptions of giftedness. Corwin Press: Thousand Oaks.

Sternberg, R.J. y Davidson, J.E. (2004) Conceptions of giftedness. Cambridge: Cambridge University Press.

Sternberg, R. J., y Davidson, J. E. (Eds.). (2005). Conceptions of giftedness. Cambridge University Press. https://doi.org/10.1017/CBO9780511610455

Suárez, J., Maiz, F., \& Meza, M. (2010). Multiple intelligences: A pedagogical innovation to enhance the process of teaching and learning. Investigación y postgrado, 25(1), 81-94. Recuperado de: https://dialnet.unirioja.es/servlet/articulo?codigo $=5330850$

Subotnik, R. F., Olszewski-Kubilius, P., \& In Worrell, F. C. (2019). The psychology of high performance: Developing human potential into domain-specific talent.

Tallent-Runnels, M., \& Tirri, K. (1998). Teachers' Attitudes Toward Gifted Education: A Cross-cultural Study. ECHA Conference. DOI: 10.1080/15332276.2004.11673039

Tannenbaum, A.J. (1991): The social psychology of giftedness. En Colangelo, N. Y Davis, G.A. (Eds): Handbook of Gifted Education. Boston: Allyn \& Bacon.

Torrego, J. C. (2012), Alumnos con altas capacidades y aprendizaje cooperativo. Un modelo de respuesta educativa. Madrid: Fundación SM.

Tourón, J. (2020). Las altas capacidades en el sistema educativo español: reflexiones sobre el concepto y la identificación. Revista De Investigación Educativa, 38(1), 15-32. DOI: https://doi.org/10.6018/rie.396781

Tourón, J., Fernández, R. y Reyero, M. (2002). Actitudes del profesorado hacia la superdotación. Implicaciones para el desarrollo de programas de formación. Faísca, 9, 95-110. Recuperado de: https://hdl.handle.net/10171/18757

UNIR (2020). Experto Universitario en Altas capacidades y Desarrollo del Talento. Recuperado el 02/04/2020 de: https:/cutt.ly/6tHWo8G

Wallace, B. y Erikson, G. (2006). Diversity in Gifted Education. International perspectives on global issues. Nueva York: Routledge.

Fecha de recepción: 9 de abril de 2020.

Fecha de revisión: 2 de mayo de 2020.

Fecha de aceptación: 14 de octubre de 2020. 